\title{
A new airborne polar Nephelometer for the measurements of optical and microphysical cloud properties. Part I: Theoretical design
}

\author{
J. F. Gayet ${ }^{1}$, O. Crépel $^{1}$, J. F. Fournol ${ }^{1}$, S. Oshchepkov ${ }^{2}$ \\ ${ }^{1}$ Laboratoire de Météorologie Physique, URA CNRS 267, Université Blaise Pascal Clermont-Ferrand, France \\ ${ }^{2}$ Institut of Physics, Minsk, Belorussia
}

Received: 18 June 1996 / Revised: 4 December 1996 / Accepted: 6 December 1996

\begin{abstract}
A new optical sensor, the airborne Polar Nephelometer, is described. The sensor is designed to measure the optical and microphysical parameters of clouds containing either water droplets or ice crystals or a mixture of these particles ranging in size from a few micrometers to about $500 \mu \mathrm{m}$ diameter. The probe measures the scattering phase function of an ensemble of cloud particles intersecting a collimated laser beam near the focal point of a paraboloïdal mirror. The light scattered from polar angles from $3.49^{\circ}$ to $169^{\circ}$ is reflected onto a circular array of 33 photodiodes. The signal processing electronics and computer storage can provide one measurement of the scattering phase function every $100 \mathrm{~ms}$ or every $0.2 \mathrm{~ms}$. The first part of the paper describes the theoretical design of a prototype version of the probe.
\end{abstract}

\section{Introduction}

Reliable cloud optical and microphysical measurements can lead to a better understanding of cloud radiation and formation processes of precipitation. We introduce here a new optical airborne instrument, the 'Polar Nephelometer', which is an attempt to improve the accuracy of measuring cloud optical and microphysical parameters over a large size range (from a few micrometers to about $500 \mu \mathrm{m}$ diameter). The Polar Nephelometer is designed to provide a direct measurement of the scattering phase function of cloud particles (water droplets and/or ice crystals). The droplet size spectra and subsequent derived quantities such as the liquid water content (LWC) and size parameters are retrieved from the measured scattering phase function by using the inversion method of Oshchepkov et al. (1993). The particle phase discrimination (water droplets/ ice particles) can be derived from the shape of the scattering phase function, and the sensitivity of the probe allows the detection of small ice crystals (typically of $5-\mu \mathrm{m}$ diameter).
Present instrumentation for measuring microphysical parameters includes the CSIRO hot-wire probe (King et al., 1978) and the Forward Scattering Spectrometer and Cloud Droplet Probes (FSSP-100, 1D \& 2D, Particle Measuring Systems, Boulder, Co., Knollenberg, 1981). This instrumentation gives limited information on cloud microphysical parameters. For instance, the CSIRO probe measures LWC with an accuracy of $15 \%$ (King et al., 1985) for droplets lower than about 50- $\mu \mathrm{m}$ diameter due to the splashing of larger droplets when they impinge the sensing hot wire of the probe. The FSSP-100 being an optical single-particle counter, the measurements' reliability is sensitive to coincidence errors which depend both on droplet concentration and airspeed. The best accuracy of LWC can be obtained by applying corrections for coincidence and dead-time losses, laser-inhomogeneity errors and time response (e.g. Dye and Baumgardner, 1984; Baumgardner et al., 1985; Brenguier, 1989). In contrast to the preceding techniques, the Polar Nephelometer does not depend on the rate (and therefore on the airspeed) at which the particles pass through the instrument. The probe looks at many droplets simultaneously in a sampling volume about 20 times larger than the sampling volume of the FSSP-100, where droplets are measured optically and individually. These differences give the Polar Nephelometer the potential of measuring particle concentration more accurately.

Despite the progress in methods of data processing, present instrumentation is crucially inadequate for reliable measurements of small ice crystals (typically smaller than $25 \mu \mathrm{m}$ ) (Platt et al., 1989; Gayet et al.,1996). The lower limit of the PMS 2D-C probes reduces their utility in assessing the radiative properties of cirrus (Arnott et al., 1994; Larsen et al., 1996). A combination of replicator probes (Strauss et al., 1995) and conventional PMS probes may be a good compromise but only scarce samplings can be carried out. Furthermore, to study the interaction between radiation and microphysics, direct measurements on the scattering properties of ice particles are strongly needed (Kinne et al., 1992). In this application the Polar Nephelometer will constitute 
an original instrument with the airborne $\Pi-N e p h e l o m e-$ ter recently developed by Lawson (1995). This later probe gives scattering phase function in $10^{\circ}$ increments for individual particles, whereas the Polar Nephelometer samples many particles with 33 angular measurements.

The Polar Nephelometer is presented in this paper. First, the principle of operation of the probe and results of the theoretical study used for the choice of the optoelectronic components will be presented. Then, results about the optimization of the optical detection and calibration procedures will be presented. Preliminary results obtained by a prototype of the probe in an open wind tunnel operated in natural cloud conditions are discussed in detail in the companion to this paper (Crépel et al., this issue).

\section{Principle of operation and design of the instrument}

\subsection{Principle of operation}

The optical scheme of the Polar Nephelometer is roughly equivalent to the photometer developed by Bartholdi et al. (1980). The cloud particles are streamed in a sampling tube equipped with a boundary-layer trap. These particles intersect a collimated laser beam at the focal point of a paraboloidal reflector (see Fig. 1). The sensitive volume of the instrument, defined by the diameter of the laser beam $(5 \mathrm{~mm})$ and the diameter of the particle inlet $(10 \mathrm{~mm})$, is relatively large $\left(\approx 0.2 \mathrm{~cm}^{3}\right)$. The scattered light is reflected onto a circular array of 33 detectors located in front of the mirror. Each detector senses signals corresponding to a range of scattering angles. The size and positions of the detectors determine the angular resolution of the differential scattering pattern. The polar scattering angle $\theta$ is $0^{\circ}$ in the forward direction with respect to the propagation vector of the incident beam.

The laser beam is provided by a high-power $(1.2 \mathrm{~W})$ multimode laser diode (Spectra Diode Labs SDL 2372

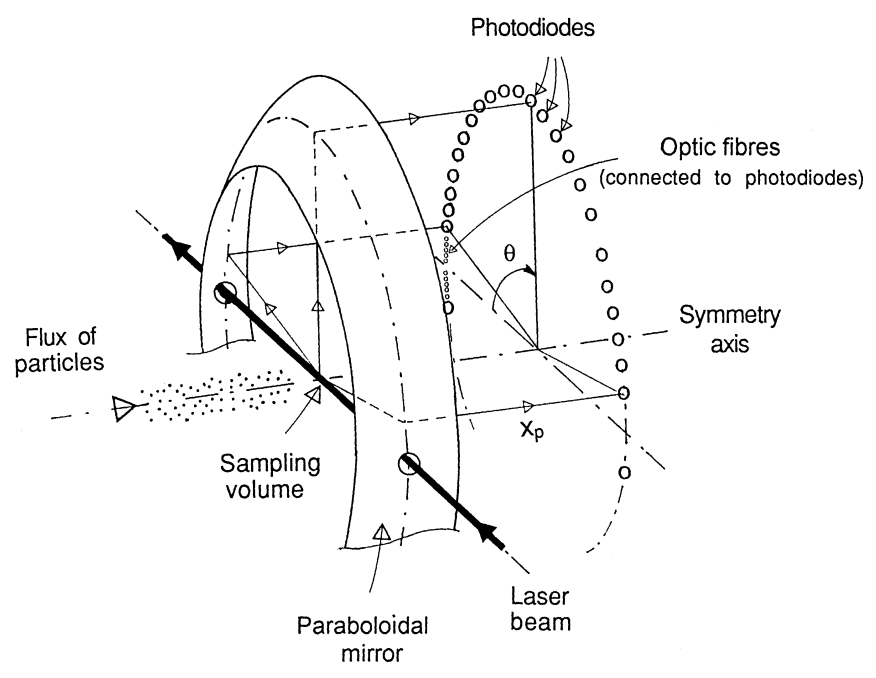

Fig. 1. Principle of measurement of the Polar Nephelometer
P3) operating at $\lambda=785 \mathrm{~nm}$. The chip temperature of the laser diode is regulated by a Peltier element in order to maintain a constant wavelength. The laser beam is routed via an optic fibre to an optical system providing a collimated beam (5 $\mathrm{mm}$ diameter) with a divergence lower than $13 \mathrm{mrad}$ and an output power of $1.0 \mathrm{~W}$ (the beam intensity can be varied by setting the laser power). The beam is not polarized in the present prototype version. The paraboloidal mirror (Melles Griot product number 02 RPM 017, focal distance: $33 \mathrm{~mm}$ ) is drilled with holes ( $8 \mathrm{~mm}$ diameter) for laser-beam entrance and exit. The reflector surface is coated with electrolytically deposited rhodium which is particularly resistant to oxidation. Rhodium has a reflectance of $80 \%$ for visible wavelengths. A light trap is placed close to the beam exit hole to avoid reflection of the incident laser beam from any surface.

\subsection{Determination of the sensitive volume and spatial resolution}

The present section concerns the determination of the sensitive volume and the spatial resolution of the nephelometer. We recall that the sensitive volume is defined as the product of the laser-beam section by the active length of the beam, that is the diameter of the inlet. The spatial resolution depends on both the sensitive volume and data acquisition procedure. In the present version of the nephelometer the data acquisition procedure simultaneously samples the 33 channel (see Sect. 3) over a quasi-instantaneous sampling duration (some $\mu \mathrm{s}$ ) and with an acquisition rate which can be selected to $10 \mathrm{~Hz}$ or to $5 \mathrm{kHz}$.

Considering the principle of the nephelometer, which is to measure the optical characteristics of a polydisperse population of particles, the number of particles present in the sensitive volume must be as high as possible, so that the measurement is statistically accurate. However, the astigmatism errors introduced by the parabolic mirror and due to the finite size of the sensitive volume have to be considered. The larger the sensitive volume, the larger the astigmatism errors.

These considerations have led to a sensitive volume equal to $0.2 \mathrm{~cm}^{3}$. This corresponds to a $10-\mathrm{mm}$-long and 5-mm-diameter beam. Considering now this sensitive volume, Table 1 shows the values of the number of particles simultaneously present in the volume, for usual cloud particle concentrations. Results in Table 1 show that for particle concentrations smaller than 5 $\mathrm{cm}^{-3}$ (for the case of cloud ice crystals), the statistical number of particles present in the sampling volume is lower than unity. This means that for these concentrations, it will be necessary to sum up several successive measurements, so that the total sampling volume contains a large enough number of particles. A total number of 100 particles appears to be large enough to average out the interference effects and, for the case of ice crystals, the orientation effects. The total sampled volume $\left(V_{\min }\right)$ containing this minimum number $\left(N_{\min }\right)$ is equal to: 
Table 1. Number of particles simultaneously present in the sampling volume and minimum flight distances (or spatial resolutions of the instrument) in order to sample 100 particles as a function of the particle concentration and acquisition rate $(f)$ assuming an airspeed $\left(V_{a}\right)$ of $100 \mathrm{~m} \mathrm{~s}^{-1}$. (*The lowest value of the spatial resolution is determined by the ratio of $V_{a}$ to $f$ )

\begin{tabular}{lcclc}
\hline Particle type & $\begin{array}{l}\text { Particle } \\
\text { concentration }\end{array}$ & $\begin{array}{l}\text { Nb of particles in } \\
\text { the sampling volume }\end{array}$ & \multicolumn{2}{c}{$\begin{array}{l}\text { Minimum flight distances (m) } \\
\text { for a sample of 100 particles } \\
10 \mathrm{~Hz}\end{array}$} \\
\hline Water droplets & $1000 \mathrm{~cm}^{-3}$ & 200 & $110^{1}(*)$ & $50^{2}$ \\
Ice crystals & $10 \mathrm{~cm}^{-3}$ & 2 & $510^{-2}(*)$ & $10^{0}$ \\
& $1000 \mathrm{l}^{-1}$ & 0.2 & $510^{6}$ & $10^{1}$ \\
\hline
\end{tabular}

$V_{\text {min }}=N_{\min } /$ Conc

with $N_{\text {min }} \sim 100$ and Conc the particle concentration, so that the minimum flight distance covered by the aircraft $\left(L_{\text {min }}\right)$ is:

$L_{\text {min }}=V_{\text {min }} / V_{s} \times V_{a} / f$,

with $V_{s}$ the sensitive volume, $V_{a}$ the airspeed and $f$ the acquisition rate $(10 \mathrm{~Hz}$ or $5 \mathrm{kHz})$. Assuming the above values of $N_{\min }$ and $V_{s}$ and an airspeed of $100 \mathrm{~m} \mathrm{~s}^{-1}$, values of $L_{\text {min }}$ are reported in Table 1 . The results show that for an acquisition rate of $5 \mathrm{kHz}$, the minimum flight distance is ranged from $2.10^{-2}$ to $10^{4} \mathrm{~m}$ for a particle concentration varying between $1000 \mathrm{~cm}^{-3}$ and $11^{-1}$. These minimum flight distances define the spatial resolution of the instrument.

We recall here that the usual PMS 1D probes (FSSP for instance, see among others Dye and Baumgardner, 1984) are optical single-particle counters. Thus, the sampling volume, which is the product of the sampling surface (typically $0.2 \mathrm{~mm}^{2}$ ) by the airspeed, is around $50 \mathrm{~cm}^{3} \mathrm{~s}^{-1}$ for an airspeed of $100 \mathrm{~m} \mathrm{~s}^{-1}$. With an acquisition rate $(f)$ of $5 \mathrm{kHz}$, the corresponding sampling volume of the Polar Nephelometer (product of $f$ by the sensitive volume) is 20 times larger than the FSSP's (1000 against $50 \mathrm{~cm}^{3} \mathrm{~s}^{-1}$ ).

\subsection{Sensitivity and dynamic range}

The Polar Nephelometer is designed to measure the scattering phase function clouds containing either water droplets or ice crystals, or a mixture of these particles, over a size range from a few micrometers to about $500 \mu \mathrm{m}$. The design and the choice of the opto-electronic components are conditioned by both the detection thresholds and the output dynamic ranges, which are themselves dependent on the sampling volume, the laserbeam power and the expected variations of the cloud microphysic parameters.

For the calculation of the sensitivity threshold and dynamic range we have considered a laser power of $1 \mathrm{~W}$ with a wavelength of $785 \mathrm{~nm}$. The lower and upper limits of detection are determined by considering one 5- $\mu \mathrm{m}$-diameter particle present in the sampling volume and one 500- $\mu \mathrm{m}$-diameter particle in the sampling volume, respectively. The subsequent light intensity $I(\theta)$ which reaches the detectors located at a polar scattering angle $(\theta)$ can be expressed by the following relationship:

$I(\theta)=P_{0} / S \cdot \sigma(\theta, d) / 4 f^{2}$,

with $P_{0}$ the incident laser power, $S$ the laser-beam crosssection, $f$ the focal length of the paraboloidal mirror and $\sigma(\theta, d)$ the scattering cross-section relative to a particle of diameter $d$. The scattering cross-section is calculated using the Mie theory assuming homogeneous spherical particles (water or ice). The scattered intensities (in $\mathrm{W} \mathrm{cm}^{-2}$ ) are shown in Fig. 2 for $\theta$ ranging from $0^{\circ}$ to $180^{\circ}$ and for the two given particle diameters. Table 2 displays the corresponding values of the scattered intensities near the forward direction $\left(5^{\circ}\right.$ : where the intensity is high) and near the side direction $\left(110^{\circ}\right.$ : lower intensity). The calculation shows that for the detection of one particle of $5 \mu \mathrm{m}$ diameter, a sensitivity of about $10^{-10} \mathrm{~W} \mathrm{~cm}^{-2}$ is needed and that a dynamic range of $10^{4}$ is enough for measuring one particle of $500 \mu \mathrm{m}$ diameter.

It is now possible to determine the measurement range in terms of particle size distribution. Figure 3 shows the isolines of liquid water content (from $10^{-4}$ to $10 \mathrm{~g} \mathrm{~m}^{-3}$ ) in a particle concentration versus mean

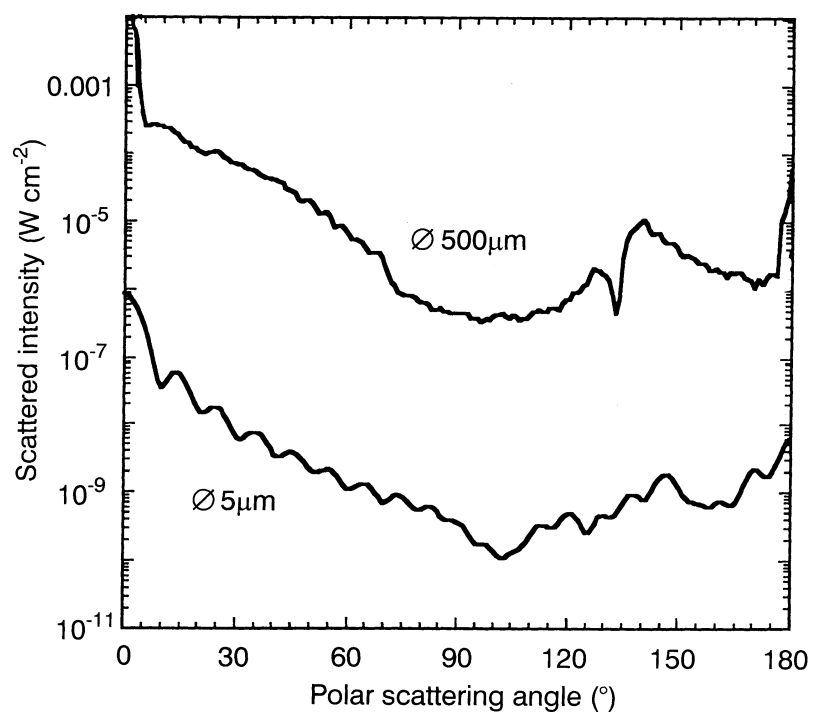

Fig. 2. Theoretical scattering phase functions (expressed in $\mathrm{W} \mathrm{cm}^{-2}$ ) for single droplet (5 and $500 \mu \mathrm{m}$ diameter, respectively) present in the sampling volume of the Polar Nephelometer 
Table 2. Values of the scattered energy $\left(\mathrm{W} \mathrm{cm}^{-2}\right)$ at $5^{\circ}$ and $110^{\circ}$ for two particle diameters

\begin{tabular}{lll}
\hline $\begin{array}{l}\text { Polar scattering } \\
\text { angles }\end{array}$ & $\begin{array}{l}\text { Particle diameter } \\
5 \mu \mathrm{m}\end{array}$ & $500 \mu \mathrm{m}$ \\
\hline $5^{\circ}$ & $3.10^{-7}$ & $1.10^{-3}$ \\
$110^{\circ}$ & $1.10^{-10}$ & $1.10^{-6}$ \\
\hline
\end{tabular}

diameter scale. The range of operation is limited by shadowed areas corresponding to the sensitivity and dynamical range already given. Figure 3 shows that the lower limit of detection corresponds approximately to a concentration of $1 \mathrm{~cm}^{-3}$ for particles of $4 \mu \mathrm{m}$ diameter, or $10 \mathrm{~cm}^{-3}$ for particles of a one micron diameter. As for the upper limit of detection, almost every kind of large particle and concentration can be measured.

\section{Design of the detector array}

The detector array consists of 33 photodiodes located on a 132-mm-diameter circle (4 times the focal length of the paraboloidal reflector, see Fig. 1). Ten detectors (located in the forward direction) consist of optical fibres which drive the light energy onto a photodiode located behind the detector array. The other 23 detectors are equipped with lenses which focus the light energy onto the active part of the photodiode. One should notice that only one half of the optical chamber is equipped with detectors (except in the forward direction, see Fig. 1) in the prototype version of the Polar Nephelometer.

\subsection{Detectors equipped with optical fibre}

The study of the information content (Crépel, 1995), with respect to the inversion method by Oshchepkov

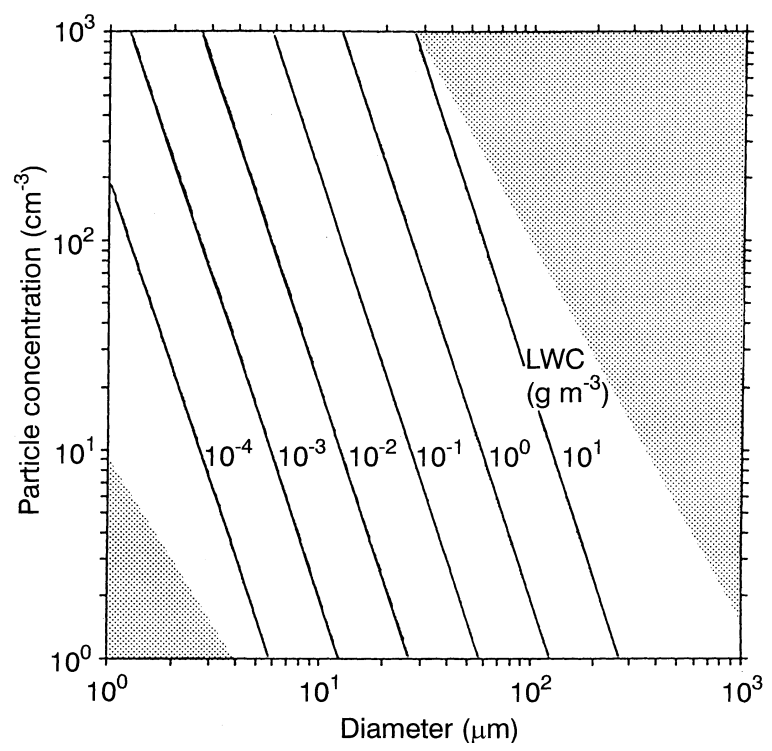

Fig. 3. Measurement range of the Polar Nephelometer versus the droplet size and concentration et al. (1993), showed that the larger the number of measurements in the forward direction, the better the accuracy of the inversion calculation. Optical fibres of $1.5 \mathrm{~mm}$ diameter (attenuation of $2.2 \mathrm{~dB} \mathrm{~m}^{-1}$ at $785 \mathrm{~nm}$ ) were selected for this application. This small size fits a reasonable number of measurements (10) in the available space and still provides a sufficient optical aperture with respect to the high values of the scattered energy in this direction. These optical fibres (around $6 \mathrm{~cm}$ long) are connected to photodiodes. Five angles are determined by each detector on both sides of the forward direction (see Fig. 4 and Table 3). The minimum angle $\left(3.49^{\circ}\right)$ is determined by the geometry defined by the diameters of both the laser and the optical fibre (see Fig. 4). The detectors located at positive and negative polar angles are staggered in order to obtain a doubling of the measurements in the forward direction determining an angular resolution of $0.79^{\circ}$. Detectors numbers 5 and 29 (see Table 3 ) are symetrically positioned $( \pm$ $10.60^{\circ}$ ) for the optical alignement settings and controls. According to the focal distance of the reflector and the optical fibre diameter the corresponding optical aperture and solid angle are, respectively, $1.30^{\circ}$ and $4.010^{-4} \mathrm{sr}$.

\subsection{Detectors equipped with lenses}

These detectors are composed of a 5-mm-diameter lens (Melles Griot product number 01 LPX 003/076, focal distance: $10.2 \mathrm{~mm}$ at $785 \mathrm{~nm}$ ) focusing the light on the active part of the photodiode (see Fig. 5). The corresponding polar angles are ranged from $15.0^{\circ}$ to $169.0^{\circ}$ with an angular resolution of $7.0^{\circ}$ (see Table 3 ). A filter is mounted in front of each lens, which has a transmittance of $92 \%$ at $785 \mathrm{~nm}$. According to the focal distance of the reflector and the lens diameter the corresponding optical aperture and solid angle are, respectively, $4.34^{\circ}$ and $4.5110^{-3} \mathrm{sr}$.

\subsection{Detection module}

The same type of detector is used for both the optical fibre and lens systems. The detectors are silicon P-I-N

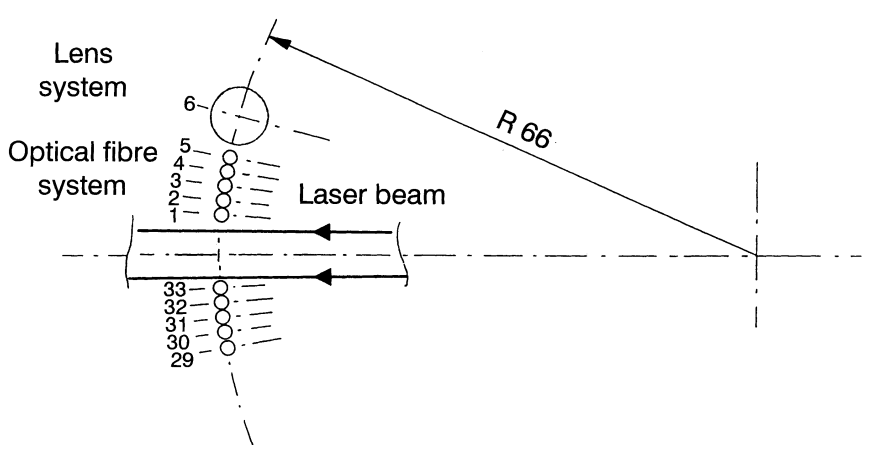

Fig. 4. Schematic diagram of the optical fibre detectors showing the laser beam, the position and numbering of the elements (see Table 3 for the corresponding values of the polar angles) 
Table 3. Subtended angles relative to each detector

\begin{tabular}{lclllr}
\hline $\begin{array}{c}\text { Fibre } \\
\text { number }\end{array}$ & $\begin{array}{l}\text { Angles } \\
\left({ }^{\circ}\right)\end{array}$ & $\begin{array}{l}\text { Photodiode } \\
\text { number }\end{array}$ & $\begin{array}{l}\text { Angles } \\
\left({ }^{\circ}\right)\end{array}$ & $\begin{array}{l}\text { Photodiode } \\
\text { number }\end{array}$ & $\begin{array}{l}\text { Angles } \\
\left({ }^{\circ}\right)\end{array}$ \\
\hline 1 & 4.28 & 6 & 15.00 & 18 & 99.00 \\
2 & 5.86 & 7 & 22.00 & 19 & 106.00 \\
3 & 7.44 & 8 & 29.00 & 20 & 113.00 \\
4 & 9.02 & 9 & 36.00 & 21 & 120.00 \\
5 & 10.60 & 10 & 43.00 & 22 & 127.00 \\
29 & -10.60 & 11 & 50.00 & 24 & 134.00 \\
30 & -8.23 & 12 & 57.00 & 25 & 14.00 \\
31 & -6.65 & 14 & 64.00 & 26 & 155.00 \\
32 & -5.07 & 15 & 71.00 & 27 & 162.00 \\
33 & -3.49 & 16 & 78.00 & 28 & 169.00 \\
& & 17 & 85.00 & & \\
\hline
\end{tabular}

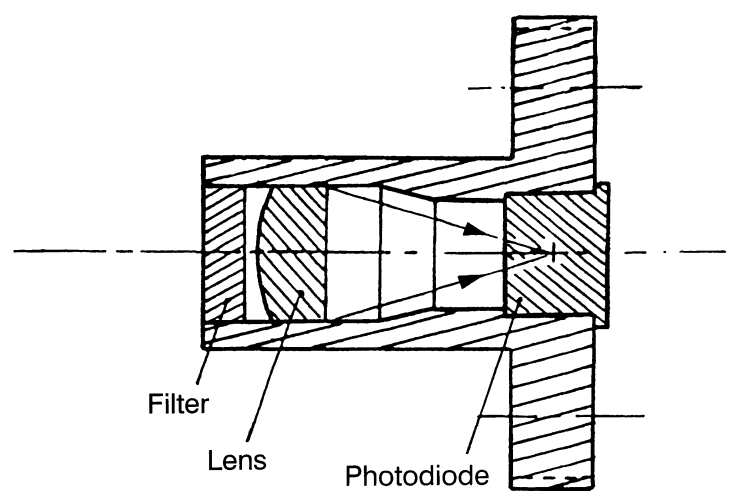

Fig. 5. Schematic diagram of the optical detector equipped with lenses

having a spectral response extending from 300 to $1150 \mathrm{~nm}$ with a peak responsivity at $890 \mathrm{~nm}$. The 4.65-mm-diameter chip was selected for this application since this small size fits a reasonable number of chips (23) into the available space and still provides sufficient active area for the threshold sensitivity determined in Sect 2.3. The $0.25-\mathrm{mm}^{2}$ active area of these chips has a sensitivity of $0.45 \mathrm{~A} \mathrm{~W}^{-1}$ at $785 \mathrm{~nm}$. The P-I-N photodiode type was selected because its responsivity is not very sensitive to ambient temperature variations. These photodiodes are operated under photovoltaïc mode (polarization voltage set to zero) for a better signal-tonoise ratio.

The outputs of the photodiode chips are configured with transimpedance amplifiers and then with logarithmic amplifiers (see Fig. 6). The transimpedance amplifiers are optimized to give a high signal-to-noise ratio with a good bandwidth. The transimpedance amplifiers' output voltage $\left(V_{1}\right)$ is given by the following relation:

$V_{1}=R_{f} I_{p}$,

with $R_{f}$ the transimpedance amplifier and $I_{p}$ the photodiode output current which is related to the optical power $(P)$ received by the photodiode:

$I_{p}=A P$,

with $A$ the photodiode response $(\approx 0.45 \mathrm{~A} / \mathrm{W})$.
The transimpedance resistance $R_{f}$ is determined for each detector such that $V_{1}$ ranges between $0.3 \mathrm{mV}$ and $3 \mathrm{~V}$, for, respectively, the lower and upper limits of detection calculated in Sect. 2.3 (Fig. 2).

The logarithmic output voltage $(V s)$ is expressed by:

$V s=K \log \left(V_{1} / 0.3 \mathrm{mV}\right)$.

A dynamic range of four orders of magnitude (see Sect. $2.3)$ sets the logarithmic gain $(K)$ to $2.5 \mathrm{~V}$ per order of magnitude for an output voltage range of $10 \mathrm{~V}$.

Determining the bandwidth of the detector array requires the calculation of the cut-off frequency of each channel. According to their specific gain, the frequency response can be calculated assuming that the transimpedance amplifier is a second-order filter (Fournol et al., 1993). The results show that most of the amplified channels (photodiodes $21 \& 22$, see Table 3 ) have their lowest cut-off frequencies at about $15 \mathrm{kHz}$. This value is compatible with an airborne use for airspeed of about $100 \mathrm{~m} \mathrm{~s}^{-1}$.

We recall that in the present version of the Nephelometer the data acquisition procedure simultaneously samples the 33 channels over a quasi-instantaneous sampling duration (some $\mu \mathrm{s})$ and every $100 \mathrm{~ms}(10 \mathrm{~Hz})$ or every $0.2 \mathrm{~ms}(5 \mathrm{kHz})$. The signals are then multiplexed to a fast 12-bit analog-to-digital converter. The digitized output is then processed by a computer for recording and subsequent real-time data processing.

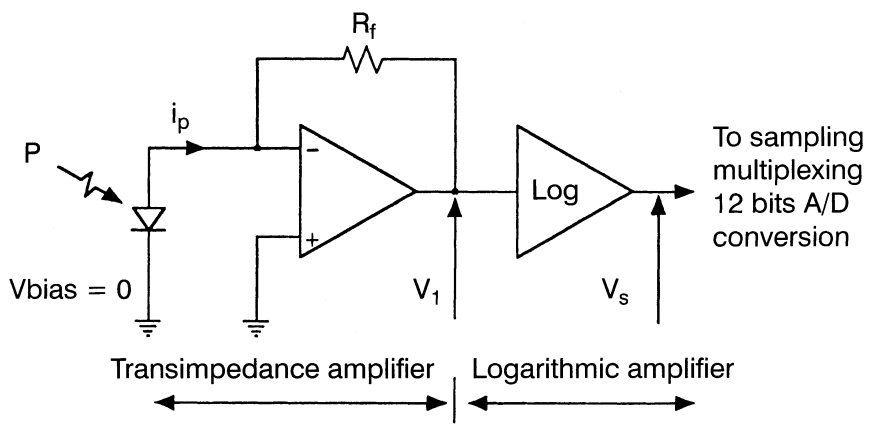

Fig. 6. Photodiode amplifier configuration 


\section{Optical design}

\subsection{Astigmatism errors}

Due to the use of a paraboloidal mirror and a finite sensitive volume, the optical system is not stigmatic. Therefore, energy scattered by a particle located outside the focal point following a given angle may not reach the corresponding detector. Numerical simulations have been made in order to estimate the subsequent errors on the measurements. These simulations (Crépel, 1995) use a ray-tracing technique for the characteristic determination of the light rays which emanate from the sampling volume and reach one detector. For one given detector, the statistical distribution of the polar scattering angle provides information on the subsequent astigmatism error. According to the sampling volume geometry, the astigmatism error is larger near the side direction $\left(\theta\right.$ close to $\left.90^{\circ}\right)$.

Figure 7 illustrates the results relative to the detector located at $92^{\circ}$ and for three distances ( $x_{p}$, cf. Fig. 1) between the focal plane of the mirror and the plane defined by the detector array. On each figure, the first curve corresponds to a perfectly stigmatic system, for which we assume a sampling volume reduced to the focal point. In this case, the angular spreading is only due to the optical aperture of the detector $\left(4.34^{\circ}\right.$, see Sect. 3.2). The second curve, for which we consider the actual sampling volume, highlights an angular spreading much larger than the stigmatic scheme in the cases (a) and (c), that is to say for distances $x_{p}$ of 10 and $100 \mathrm{~mm}$, respectively. For the case (b), which is characterized by a distance of $65 \mathrm{~mm}$, the results show that the two curves are quite similar, indicating that the astigmatism errors are considerably reduced at this distance. Consequently, the plane of the detector array has been positioned at $x_{p}=65 \mathrm{~mm}$.

\subsection{Reduction of the optical interferences}

It is imperative to minimize the spurious reflected light from both the incident illumination and the scattered radiation, and also to reduce the effects of outside light which may enter the optical chamber. As a matter of fact, this spurious light can be comparable in intensity to the scattering signal, particularly for the side and back angles characterized by the lowest scattering intensities (see Fig. 2). One solution consists in reducing the coneaperture of the detectors so that they can only be reached by the rays coming from the sampling volume.

The photodiodes equipped with lenses have therefore been positioned at the bottom of sinks (see Fig. 8) which have an optimized depth. This depth was numerically computed using the same method of ray tracing presented in the previous section (Crépel, 1995). The results in Fig. 8 exemplify the rays emanating from the sampling volume and reaching the aperture of the photodiode located at $90^{\circ}$. The depth of the sink (35 $\mathrm{mm}$ ) has therefore been fitted to the corresponding ray envelope.
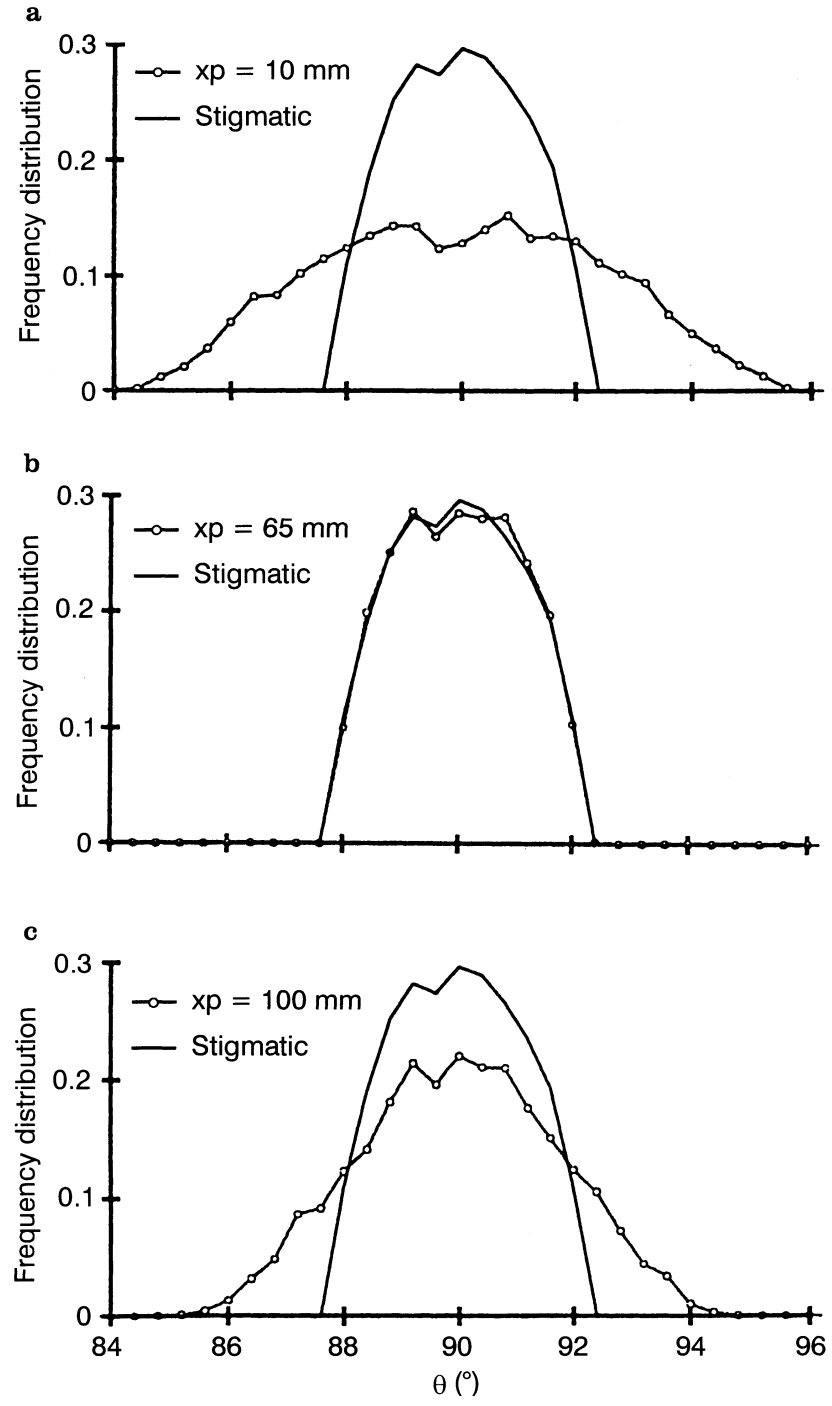

Fig. 7a-c. Statistical distribution (dotted curves) of the polar scattering angle for three values of the distance $x_{p}$ (see Fig. 1) between the focal plane of the mirror and the plane defined by the detector array. The solid curve corresponds to a perfectly stigmatic system

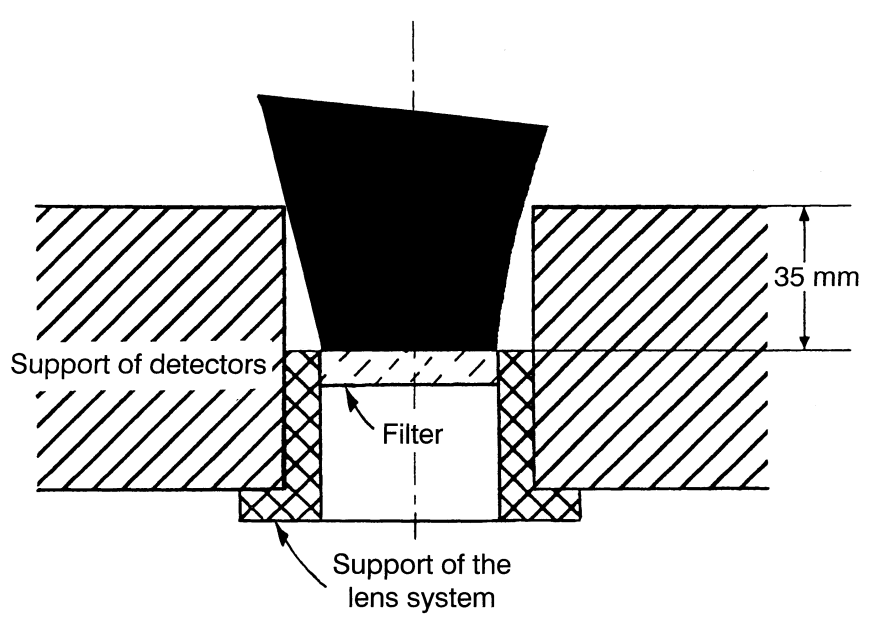

Fig. 8. Schematic diagram (not in scale) of the sink-positioned detector and cone-acceptance of the scattered beams emanating from the sampling volume 
As for the channels equipped with optical fibre, it must be remembered that the active area of the detector is defined by the cross-section of the fibre which is introduced into the hole drilled into the detector array. The sink will therefore occult part of the scattered light. Nevertheless, it is imperative to reduce the aperture of the fibres because of their large numerical aperture $\left(30^{\circ}\right)$. Calculations and laboratory experiments have shown that a sink depth of $5 \mathrm{~mm}$ noticeably reduces spurious reflected light and leads to a sampling volume occulation of only $15 \%$.

Furthermore, all the elements inside the optical chamber (see Fig. 1) are black coated in order to minimize the diffuse or specular reflected light that may contaminate the detector responses.

\section{Calibration procedure}

The calibration procedure of the different channels consists in the experimental determination of the relationship between the voltage output and the light power reaching the photodiode $P(\theta)$. Then the volume scattering cross-section $\varphi(\theta)$ can be calculated with the following relation:

$\varphi(\theta)=P(\theta) /(P o L d \Omega)$,

with $P_{0}$ the incident laser power, $L$ the length of the sampling volume and $d \Omega$ the optical aperture of the detector.

The experimental procedure uses a Light Emitting Diode (ABB HAFO product ref.: 1A208 monitored by a photodiode P-I-N ref.: 1A360M). This LED works at a wavelength of $800 \mathrm{~nm}$ and has a large dynamical range which covers the power input ranges of all the detectors. The experimental procedure requires two steps: the first aims at the determination of the LED calibration and the second at the detector calibration.

\subsection{LED calibration}

The LED calibration is realized by using a radiometer set at a distance from the LED such that the solid angles are equal to those subtended either by the lenses or the optical fibre systems (see Sect. 3). This distance is determined according to the values of the solid angles and the active area of the radiometer (see Fig. 9). Figure 10 illustrates the results of the LED calibration which are relative to the lens system, that is to say the measured light power (expressed in $\mathrm{nW}$ ) as a function of the current supply. The parameterized curve represents the best fit of the data points.

\subsection{Detector calibration}

An adapted experimental system was designed in order to proceed to the detector calibration. The active part of the LED is set at the focal point of the mirror and can be successively oriented along the optical axis of each

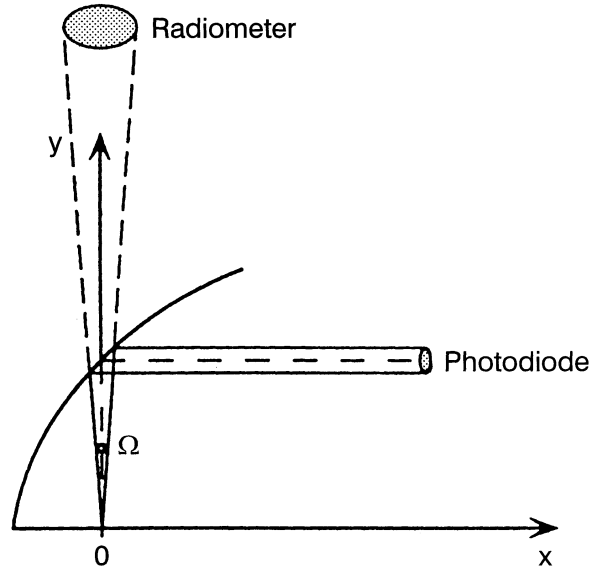

Fig. 9. Schematic diagram of the detector calibration

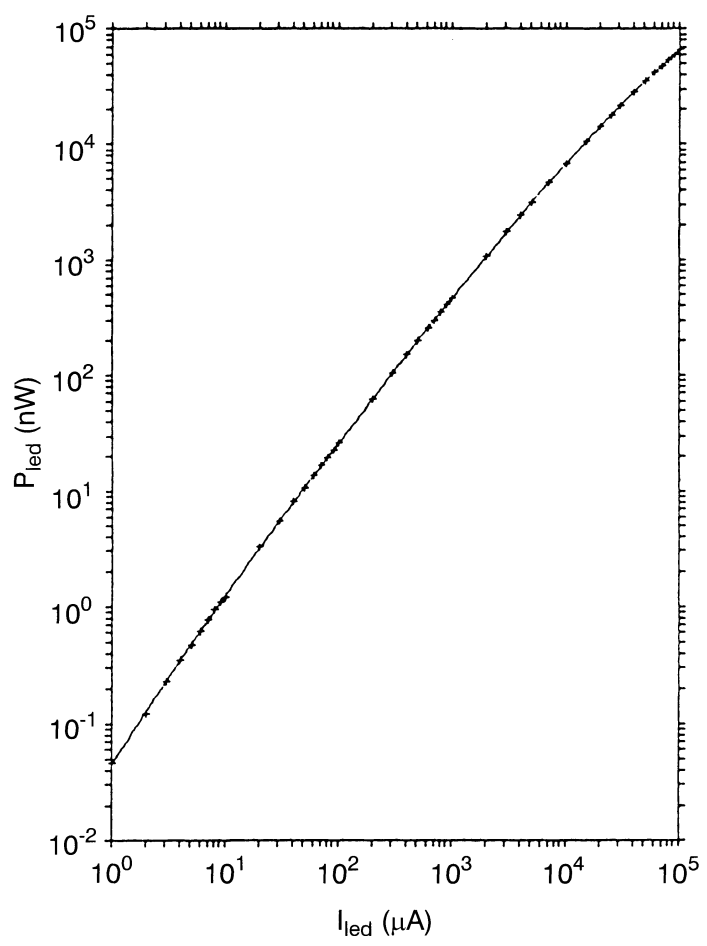

Fig. 10. Results of the LED calibration. The curve corresponds to the best fit of the data points

detector. Then for different settings of the current supply (and therefore to the input light power values following the relationship already determined) the outputs of both the transimpedance amplifier $\left(V_{1}\right)$ and logarithmic amplifier $(V S)$ have been measured. Therefore, the relationship between the input light power and the output voltage $(V s)$ can be deduced. Figures 11 and 12 illustrate the results relative, respectively, to detector number $12\left(\theta=57^{\circ}\right)$ equipped with lense and to detector number $4\left(\theta=9.02^{\circ}\right)$ equipped with optical fibre. The non-linearity (in log-scale representation) of the response curves for the lowest output voltage values are due to offset compensation set to reduce both the electronic noise for the channels equipped with lenses 

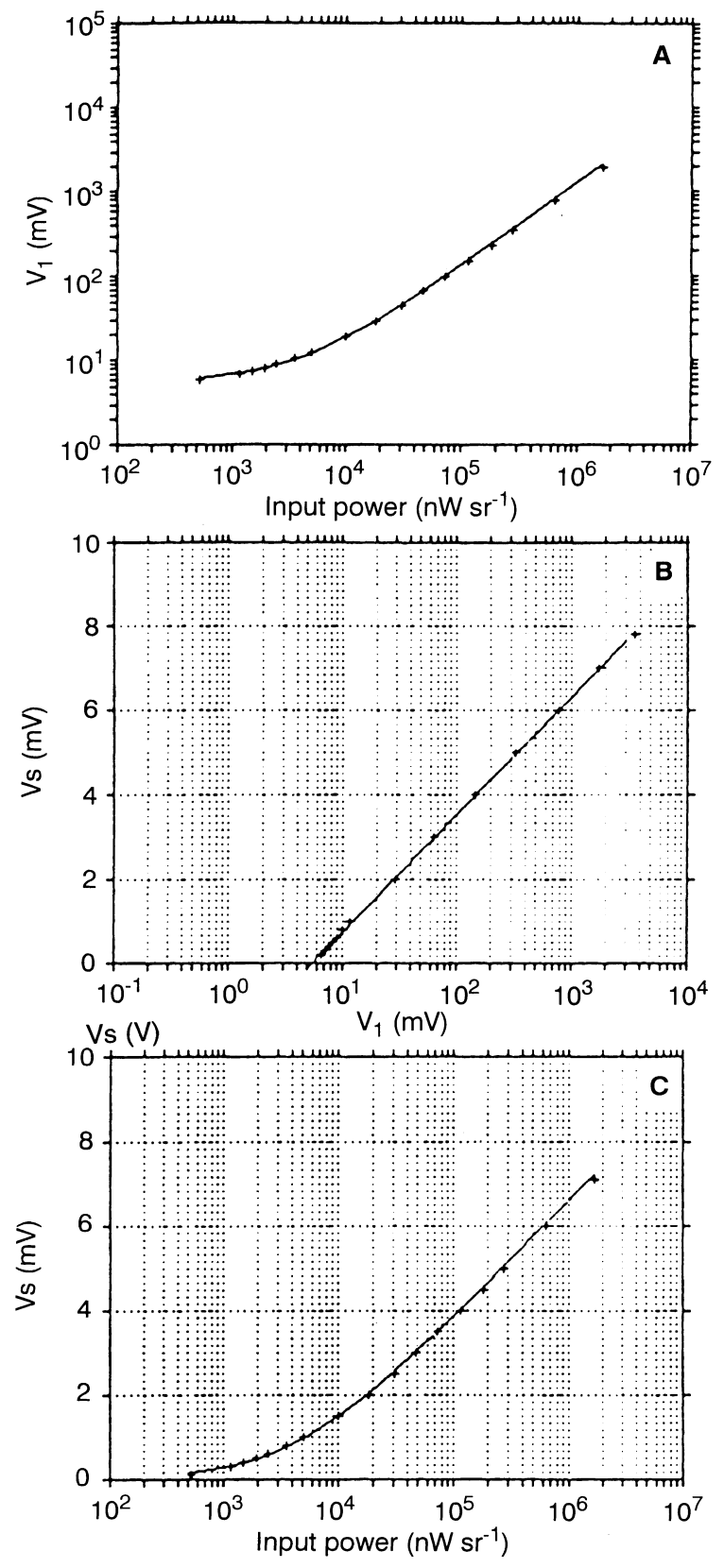

Fig. 11A-C. Results of the calibration of detector $12\left(\theta=57^{\circ}\right)$ equipped with lens system. A Transfer function of the transimpedance amplifier. B Transfer function of the logarithmic amplifier. C Calibration curve of the considered detector

and spurious light diffraction which affects the channels equipped with optical fibres (Crépel, 1995).

\section{Conclusions}

This paper describes the theoretical operation of a new airborne sensor. It is designed to provide direct measurement of the scattering phase function of cloud particles. The principle of operation of the probe and results of the theoretical study used for the selection of the optoelectronic components are discussed. Results concerning the optimization of the optical detection and
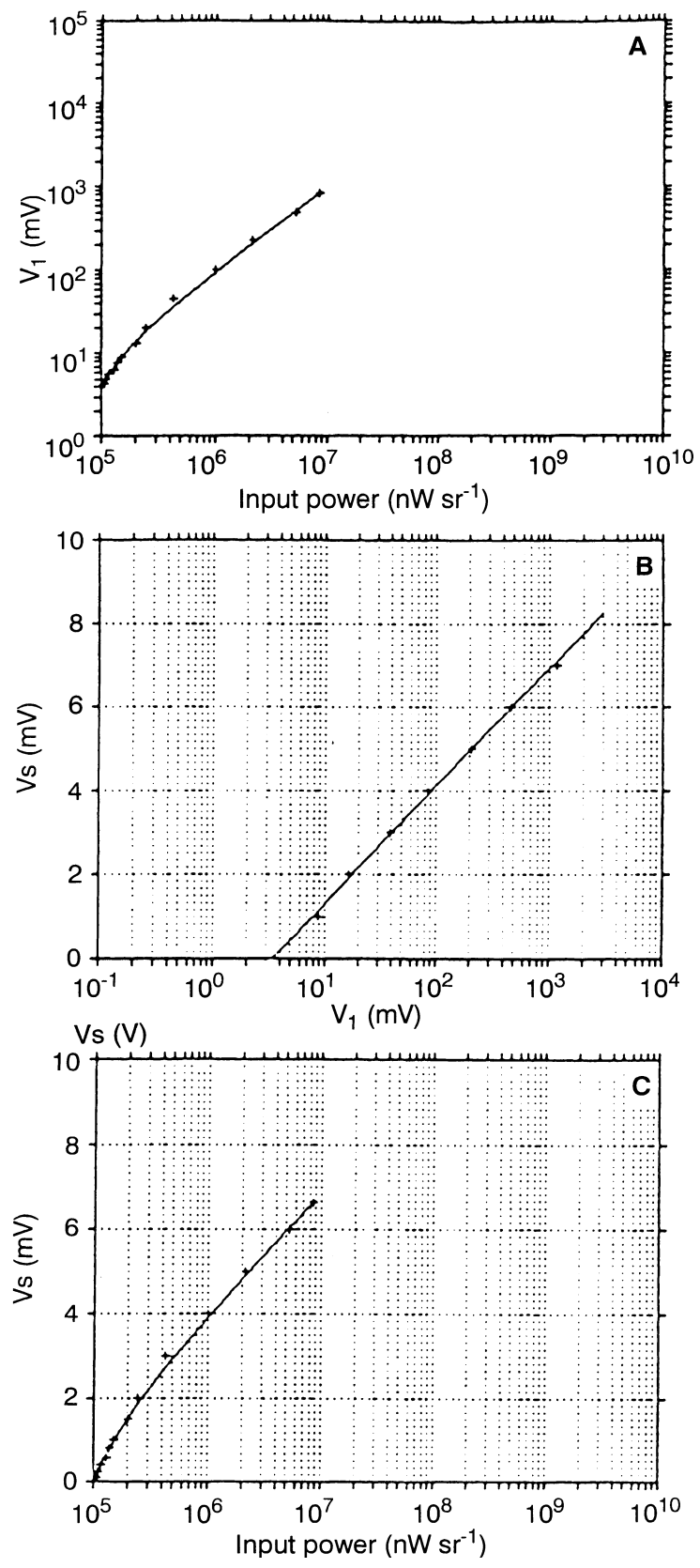

Fig. 12A-C. Same as Fig. 10. Results of the calibration of the detector $4\left(\theta=9.02^{\circ}\right)$ equipped with optical fibre system

calibration procedures are also presented. Primary results obtained with a prototype of the probe in an open wind tunnel operated in natural cloud conditions are discussed in detail in the companion to this paper (Crépel et al., this issue).

Acknowledgements. This work was supported by grants from DRET n 9134118 and $n^{\circ} 9434050$. We are very grateful to Professor Harumi Isaka, Director of the LaMP, for his helpful suggestions and discussions during the development of the project. We thank Laurent Cortes for his technical assistance. Jean-Louis Brenguier and anonymous reviewer made important comments that strengthened the manuscript.

Topical Editor L. Eymard thanks J.-L. Brenguier and another referee for their help in evaluating this paper. 


\section{References}

Arnott, W. P., Y. Y. Dong, and J. Hallett, Role of small ice crystals in radiative properties of cirrus. A case study, FIRE II, November 22, 1991, J. Geophys. Res., 99, 1371-1381, 1994.

Bartholdi, M., G. C. Salzman, R. D. Hiebert, and M. Kerker, Differential light scattering photometer for rapid analysis of single particles in flow, Appl. Optics, 19, 1573-1581, 1980.

Baumgardner, D., W. Strapp, and J. E. Dye, Evaluation of the Forward Scattering Spectrometer Probe. Part II: Corrections for coïncidence and dead-time losses, J. Atmos. Ocean. Technol. 2, 626-632, 1985.

Brenguier, J-L., Coincidence and dead-time corrections for particle counters. Part II: High concentration measurements with an FSSP, J. Atmos, Ocean Technol., 6, 585-598, 1989.

Crépel, O., Conception et mise on oeuvre d'un Néphélomètre polaire pour la mesure des paramètres optiques et microphysiques des nuages, Ph. D. Université Blaise Pascal, 1995.

Crépel, O., J-F Gayet, J-F Fournol, and S. Oshchepkov, A new airborne Polar Nephelometer for the measurements of optical and microphysical cloud properties, Part II: Performance in natural cloud conditions, this issue, 1996.

Dye, J. E., and D. Baumgardner, Evaluation of the forward scattering spectrometer probe. Part I: Electronic and optical studies, J. Atmos. Ocean. Technol., 2, 329-344, 1984.

Fournol, J-F, Crépel, O., and J-F Gayet, Projet de Néphélomètre aéroporté: Etude de la détection, Note $O P G C n^{\circ} 120$, Université Blaise Pascal, 1993.

Gayet, J-F, G. Febvre, and H. Larsen, On the reliability of the PMS FSSP probe in the presence of small ice crystals, J. Atmos. Ocean. Technol., 13, 1300-1310, 1996.
King, W. D., D. A. Parkin, and R. J. Handsworth, Hot-wire water device having fully calculable response characteristics, J. Appl. Meteorol., 17, 1809-1813, 1978.

King, W. D., J. E. Dye, J. W. Strapp, D. Baumgardner, and D. Huffman, Icing wind tunnel tests on the CSIRO liquid water probe, J. Atmos. Ocean. Technol., 2, 340-352, 1985.

Kinne S., T. P. Ackerman, A. J. Heymsfield, F. P. J. Valero, K. Sassen, and J. D. Spinhirne, Cirrus microphysics and radiative transfer: Cloud field study on 28 October 1986, Mon. Weather Rev., 20, 661-684, 1992.

Knollenberg, R. G., Techniques for probing cloud microstructure In Clouds, Their formation, Optical properties and Effects Eds. P. V. Hobbs and A. Deepak, Academic Press, New York, pp. 15-92, 1981.

Larsen, H. R., J-F. Gayet, H. Chepfer, G. Brogniez, and G. Febvre, Measurement errors in cirrus cloud microphysical properties, submitted to Ann. Geophysicae, 1996.

Lawson, R. P., Digital holographic measurements of cloud particles, Conf. on Cloud Physics, Dallas, Am. Meteor. Soc., 90-95, 1995.

Oshchepkov, S. L., O. V. Dubovik, and T. V. Lapyonok, A method of numerical solution of line-inverse problem with log-normal noise distribution: the estimation of aerosol size distribution, IRS-92 Current Problems in Atmospheric Radiation, Deepak, Hampton, Va., 334-337, 1993.

Platt, C. M. R., J. D. Spinhirne, and W. D. Hart, Optical and microphysical properties of a cold cirrus cloud: evidence for regions of small ice particles, J. Geophys. Res., 94, 151-164, 1989.

Strauss, B., J. Hallett, and P. Wendling, Small crystals in cirrus clouds, 7th EUCREX Workshop, 13-15 september 1994, Villeneuve d'Ascq, France, Ed. G. Brogniez 1995. 